\title{
Seniority zero pair coupled cluster doubles theory
}

\author{
Tamar Stein, ${ }^{1}$ Thomas M. Henderson, ${ }^{1,2}$ and Gustavo E. Scuseria ${ }^{1,2}$ \\ ${ }^{1}$ Department of Chemistry, Rice University, Houston, Texas 77251, USA \\ ${ }^{2}$ Department of Physics and Astronomy, Rice University, Houston, Texas 77251, USA
}

(Received 27 March 2014; accepted 15 May 2014; published online 6 June 2014)

\begin{abstract}
Coupled cluster theory with single and double excitations accurately describes weak electron correlation but is known to fail in cases of strong static correlation. Fascinatingly, however, pair coupled cluster doubles (p-CCD), a simplified version of the theory limited to pair excitations that preserve the seniority of the reference determinant (i.e., the number of unpaired electrons), has mean field computational cost and is an excellent approximation to the full configuration interaction (FCI) of the paired space provided that the orbital basis defining the pairing scheme is adequately optimized. In previous work, we have shown that optimization of the pairing scheme in the seniority zero FCI leads to a very accurate description of static correlation. The same conclusion extends to p-CCD if the orbitals are optimized to make the p-CCD energy stationary. We here demonstrate these results with numerous examples. We also explore the contributions of different seniority sectors to the coupled cluster doubles (CCD) correlation energy using different orbital bases. We consider both Hartree-Fock and Brueckner orbitals, and the role of orbital localization. We show how one can pair the orbitals so that the role of the Brueckner orbitals at the CCD level is retained at the p-CCD level. Moreover, we explore ways of extending CCD to accurately describe strongly correlated systems. ()
\end{abstract} 2014 AIP Publishing LLC. [http://dx.doi.org/10.1063/1.4880819]

\section{INTRODUCTION}

The electronic correlation energy is usually divided into two parts. Dynamic correlation accounts for the mutual movements of the electrons and is dominant in cases where a single Slater determinant serves as a good approximation to the wave function. In such cases, modern methods in quantum chemistry, such as coupled cluster with single and double excitations (CCSD) ${ }^{1}$ and CCSD with perturbative triple excitations $[\operatorname{CCSD}(\mathrm{T})]^{2}{ }^{2}$ can very accurately describe dynamic electron correlation, ${ }^{3,4}$ particularly when using explicitly correlated methods. ${ }^{5}$ Static or non-dynamic electron correlation is important in cases with a near-degenerate ground state, where more than a single determinant is needed for the qualitative description of the system. Such situations arise, for example, in bond-breaking processes, the description of radicals, transition metal complexes, superconductivity, and other properties of materials. For such cases, a single Slater determinant gives qualitatively wrong results, and methods such as CCSD and $\operatorname{CCSD}(\mathrm{T})$ break down. In order to obtain the correct description, one needs to take into account higher excitations, which of course are much more computationally demanding. ${ }^{6-8}$ Static correlation can be described using methods such as CASSCF $^{9}$ and full optimized reaction space. ${ }^{10}$ The scaling of the above methods is exponential and, thus, they are only applicable to small molecules. Projected Hartree-Fock can capture strong or static correlation effects with Hartree-Fock (HF) scaling and serves as an important step forward, but unfortunately, this method is not size extensive. ${ }^{11-13}$

We should mention that there are many attempts in the literature to describe static correlation using some variant of CC. ${ }^{14,15}$ Some examples are multireference CC which includes several different approaches, ${ }^{16-23}$ variational CC methods which, however, are only applicable to small systems, ${ }^{24,25}$ or the CC valence bond (CCVB) method, and its variant CCVB-SD, that improve upon the restricted CCSD description for strongly correlated systems..$^{26,27}$

Bytautas et al. have shown that the low seniority sectors of Hilbert space are most important for the description of static correlation. ${ }^{28}$ The seniority number is the number of unpaired electrons in a determinant. A seniority zero wave function describes a system in which all electrons are paired; in seniority two, one electron pair is broken and we have two unpaired electrons; in seniority four, two pairs are broken and we have four unpaired electrons, etc. The correct behavior at dissociation can often be taken into account with a seniority zero wave function alone. ${ }^{28}$ The concept of seniority has a long history in nuclear physics. ${ }^{29}$

In this work we use a closed-shell reference determinant, i.e., we pair each $\alpha$ electron with a $\beta$ electron in a spatial orbital. One must specify the identity of the underlying spatial orbitals which constitute this determinant. For example, in a diatomic molecule one could use delocalized molecular orbitals or localized atomic-like orbitals; low-seniority excitations out of these two orbital sets will yield different results even though the underlying reference determinant is not changed. One should, therefore, optimize the pairing scheme (that is, the identity of these paired molecular orbitals) in order to produce a well-defined method. $^{28,30}$

At the coupled cluster level, the simplest seniority zero wave function is given by pair coupled cluster doubles (p$\mathrm{CCD}$ ), i.e., coupled cluster doubles (CCD) with only the pair excitations taken into account, or, in other words, CCD 
restricted to preserve the seniority of the reference determinant. In a series of recent papers, Ayers and co-workers ${ }^{30-35}$ have shown that this wave function belongs to a general family of approximate antisymmetrized product of interacting geminals wave functions (APIG). ${ }^{36}$ They refer to this wave function as the antisymmetrized product of one-referenceorbital geminals (AP1roG), to indicate that each geminal contains one distinct orbital pair with respect to the reference determinant. One can show that the amplitude equations for AP1roG, which are obtained by solving projected Schrodinger equations, ${ }^{26,37}$ and the amplitude equations for p-CCD, which are obtained by solving the CC equations with the similarity transformed Hamiltonian, are in fact identical, and thus AP1roG and p-CCD are exactly the same method. Limacher et al. ${ }^{34}$ have shown that if an optimal pairing scheme is used, p-CCD can very accurately reproduce results obtained using the seniority zero full configuration interaction (FCI), which they call doubly occupied CI (DOCI). This is quite remarkable, since the scaling of seniority zero FCI is exponential, but one can get essentially the same results using p-CCD, which scales as $N^{3}$, though with an $N^{5}$ integral transformation from the atomic to molecular orbital basis, where $N$ is proportional to the number of basis functions. In the work of Limacher et al., ${ }^{34}$ the optimal pairing scheme was obtained by optimizing the orbitals at the DOCI level. The optimal pairing scheme can more efficiently be found at the p-CCD level by making the CC energy stationary with respect to orbital rotation parameters, ${ }^{38}$ using the $\mathrm{p}-\mathrm{CCD}-\Lambda$ functional defined below in analogy with CCD$\Lambda .{ }^{39,40}$ This gives us a well-defined orbital set, and thus p-CCD is itself well defined, in principle, although multiple stationary points are possible in practice. In addition to the fact that p-CCD can capture an important part of the static correlation at low computational cost, the method is size consistent with the optimal pairing scheme provided that one allows for broken symmetry orbitals, ${ }^{28}$ which makes it very appealing for many applications studying large strongly correlated systems. ${ }^{35}$

In this study, we focus on the Hubbard model, which is dominated by strong correlation for large values of $U / t$ as will be discussed below. We start by demonstrating that $\mathrm{p}-\mathrm{CCD}$ with orbital optimization gives results which are similar to seniority zero FCI in Sec. III. We then study the performance of p-CCD in different orbital sets, and study the contributions of different seniorities at the CCD level in these sets. By obtaining insight into the role of different orbital sets, we show that in analogy to CCD, where the use of Brueckner orbitals gives results which are similar to orbital optimization, one can get similar results to orbital-optimized p-CCD (oo-p-CCD) by using Brueckner orbitals together with orbital localization. Moreover, as p-CCD captures the important static correlation effects, we will try to achieve a better balance between static and dynamic correlation at the CCD and CCSD level by first solving for the seniority zero sector (that is, solving the $\mathrm{p}$ CCD equations) and subsequently solving the additional seniority sectors in CCD with seniority zero amplitudes fixed. Using this approach, we are able to extend the range of $U / t$ for which CCD can still describe the Hubbard Hamiltonian without breaking down.

\section{HUBBARD HAMILTONIAN}

In this study, we focus on the Hubbard model Hamiltonian. ${ }^{41}$ This model is widely studied in condensed matter physics and known to contain strong correlation effects for large values of Ult. The Hamiltonian is given by

$$
H=-t \sum_{j, \sigma}\left(c_{j+1, \sigma}^{\dagger} c_{j, \sigma}+c_{j, \sigma}^{\dagger} c_{j+1, \sigma}\right)+U \sum_{j} n_{j \uparrow} n_{j \downarrow},
$$

where $c_{j, \sigma}^{\dagger}$ and $c_{j, \sigma}$ are fermionic creation and annihilation operators with spin $\sigma$ on site $j$, and $n_{j, \sigma}=c_{j, \sigma}^{\dagger} c_{j \sigma}$. The first term is the hopping term, and represents the kinetic energy of electrons hopping between two adjacent sites. The second term represents the on-site repulsion $(U>0)$ between electrons. The ratio between $U$ and $t$ dictates the strength of the correlation of the system. For large values of $U / t$, the dominant part is the repulsion between the electrons and the model becomes strongly correlated. The Hubbard model, crudely, is analogous to a minimum basis chain of hydrogen atoms, with increasing $U / t$ tantamount to increasing the separation between the atoms. The model is well studied, and in one dimension exact results are available; for the 1D Hubbard model with periodic boundary conditions, exact results are known from the Bethe ansatz solution, ${ }^{42,43}$ and for the 1D Hubbard model with open boundary conditions, one can use results from Density Matrix Renormalization Group (DMRG), ${ }^{44,45}$ as DMRG is essentially exact for 1D systems. The Hubbard model is, thus, a natural choice for studying the performance of p-CCD; on the one hand, we can model a scenario of high relevance to chemistry such as bond breaking but on the other hand, we do not need to deal with large dynamic correlation blurring the important effects. Throughout this paper, we will work on 1D Hubbard chains with open boundary conditions, and compare our results to DMRG results obtained with the open-source ALPS software. ${ }^{46,47}$

\section{ORBITAL OPTIMIZED p-CCD}

A well-defined set of orbitals can be obtained by making the functional

$$
E_{p-C C D}=\left\langle 0\left|\left(1+\Lambda_{P}\right) e^{-T_{P}} H e^{T_{P}}\right| 0\right\rangle
$$

stationary with respect to orbital rotations. Here, $T_{P}$ is the pair excitation operator and $\Lambda_{P}$ is the pair de-excitation operator. We follow the procedure introduced by Bozkaya et al. for $\mathrm{CCD},{ }^{40}$ which is equivalent to the coupled cluster orbital optimization previously formulated. ${ }^{38}$ The p-CCD amplitude equations are obtained from the demand that $E_{p-C C D}$ is stationary with respect to the $\lambda$ amplitudes, and an equation for the $\lambda$ amplitudes is obtained by demanding that $E_{p-C C D}$ is stationary with respect to the $t$ amplitudes. Variations in the orbitals are expressed via the unitary exponential operator $e^{K}$, where $K$ is

$$
K=\sum_{p, q} \kappa_{p q}\left(c_{p}^{\dagger} c_{q}-c_{q}^{\dagger} c_{p}\right) .
$$

The Lagrangian as a function of $\kappa$ is

$$
\tilde{E}_{p-C C D}(\kappa)=\left\langle 0\left|\left(1+\Lambda_{P}\right) e^{-T_{P}} e^{-K} H e^{K} e^{T_{P}}\right| 0\right\rangle .
$$


TABLE I. Comparison between oo-p-CCD and optimally paired DOCI for 6-, 8-, 10-, and 14-site Hubbard chains at half filling.

\begin{tabular}{|c|c|c|c|c|c|c|c|c|}
\hline \multirow[b]{2}{*}{$U$} & \multicolumn{2}{|c|}{6 sites $(\%)$} & \multicolumn{2}{|c|}{8 sites $(\%)$} & \multicolumn{2}{|c|}{10 sites $(\%)$} & \multicolumn{2}{|c|}{14 sites $(\%)$} \\
\hline & oo-p-CCD & DOCI & oo-p-CCD & DOCI & oo-p-CCD & DOCI & oo-p-CCD & DOCI \\
\hline 2 & 85.46 & 85.48 & 83.41 & 83.42 & 81.99 & 82.00 & 80.09 & 80.10 \\
\hline 5 & 92.02 & 92.02 & 90.74 & 90.75 & 89.91 & 89.92 & 88.89 & 88.90 \\
\hline 9 & 96.97 & 96.97 & 96.51 & 96.51 & 96.23 & 96.23 & 95.88 & 95.88 \\
\hline
\end{tabular}

The first and second derivatives of the energy with respect to $\kappa$ at $\kappa=0$ are

$$
\begin{gathered}
w_{p q}=\left.\frac{\partial \tilde{E}}{\partial \kappa_{p q}}\right|_{\kappa=0}, \\
A_{p q, r s}=\left.\frac{\partial^{2} \tilde{E}}{\partial \kappa_{p q} \partial \kappa_{r s}}\right|_{\kappa=0} .
\end{gathered}
$$

After expanding the energy up to second order, minimization of the energy with respect to $\kappa$ yields

$$
\kappa=-A^{-1} w .
$$

The exponential operator $e^{K}$ is expanded up to second order, and the resulting rotation matrix is orthogonalized via a modified Gram-Schmidt procedure. For more details we refer the reader to Ref. 40 . We have used the equations given in Refs. 40, 48, and 49, and eliminated all $t$ and $\lambda$ amplitudes with non-zero seniority.

We compare the percentage of correlation energies obtained using DOCI and oo-p-CCD for 6-, 8-, 10-, and 14-site Hubbard chains at half filling in Table I. For the remainder of this paper, DOCI refers to DOCI with DOCI optimal orbitals; i.e., the DOCI orbitals were optimized to obtain the lowest DOCI energy. Furthermore, we refer to the parameter $U$ as the correlation strength (instead of $U / t$ ), as we set $t=1$. One can see that oo-p-CCD very accurately reproduces the results obtained from DOCI.

As $U$ gets larger, the system is more strongly correlated, and the performance of DOCI and oo-p-CCD improves, so that at $U=9$, around $96 \%$ of the correlation energy is recovered. We note again the resemblance between the two methods; Limacher et al. ${ }^{34}$ have shown that p-CCD with orbitals optimized for DOCI gives results that are similar to DOCI. ${ }^{34}$ Here, we see that we can achieve the same result with orbitals optimized at the p-CCD level without the need for the DOCI orbitals. We further demonstrate the ability of oo-p-CCD to describe strongly correlated systems by considering chains of up to 20 sites and by doping the system with holes, as shown in Table II.

TABLE II. Percentage of correlation energy recovered by oo-p-CCD for 20 -site chains with different fillings.

\begin{tabular}{lccc}
\hline \hline$U$ & 20 sites/20 e (\%) & 20 sites/16 e (\%) & 20 sites/12 e (\%) \\
\hline 2 & 78.38 & 72.42 & 73.74 \\
5 & 88.07 & 82.18 & 82.65 \\
9 & 95.6 & & 91.61 \\
\hline \hline
\end{tabular}

Again, we see that for more strongly correlated systems (i.e., larger $U$ ), we recover a larger portion of the correlation energy. The percentage of the correlation energy recovered for the doped systems is smaller than the percentage of correlation energy recovered at half filling. We note that for the 20 -site case, the system at half filling is more strongly correlated than the doped systems. This is evident from the fact that while RHF diverges from exact solution in all cases, this divergence is moderated for the doped systems. This demonstrates again that oo-p-CCD performs better for more strongly correlated systems.

We conclude from these results that, remarkably, static correlation can be captured by a simplified theory that uses only the lowest seniority sector in CCD, even though it is well known that CCD cannot generally describe strongly correlated systems. As a model system to demonstrate this, we choose a 6-site Hubbard chain at half filling. Figure 1 shows the performance of different methods in describing this system. It is clear from the figure that RHF is not a suitable starting point in this case, and thus the failure of CCSD and CCD is not surprising. Both CCD and CCSD start to overcorrelate near $U=5$ and catastrophically fail as $U$ gets larger. DOCI, however, captures a significant amount of the correlation and gives a qualitatively correct description provided that the optimal orbitals are used. Using oo-p-CCD, we obtain results which are indistinguishable from DOCI.

It is important to note that although p-CCD is not invariant to orbital rotations, we can find a transformation that will give us the optimal pairing. Within this pairing scheme, the method is completely well defined (although one cannot disregard the potential existence of multiple solutions).

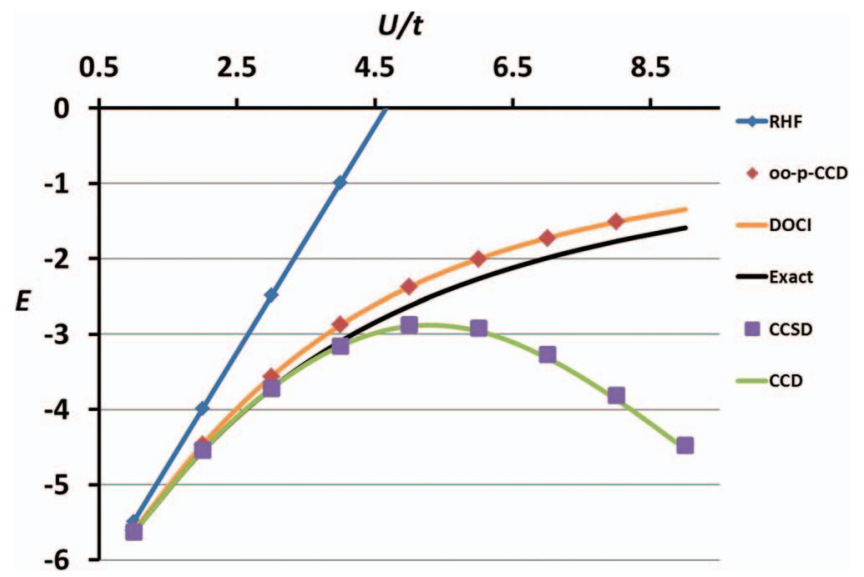

FIG. 1. Performance of different methods for the description of the 6-site Hubbard chain at half filling. 


\section{SENIORITY CONTRIBUTIONS TO THE CCD ENERGY WITHIN DIFFERENT ORBITAL BASES - THE ROLE OF ORBITAL LOCALIZATION}

We have demonstrated that orbital-optimized p-CCD gives a well-defined method which, like DOCI, can capture a significant amount of the static correlation energy. It is interesting at this point to study the performance of p-CCD when we do not optimize the orbital basis. Figure 2 shows the performance of p-CCD with different orbital bases.

As one can see from the plot, the results are quite poor when pairing canonical RHF orbitals. However, the results improve dramatically when we introduce EdmistonRuedenberg localization ${ }^{50}$ after which each of the occupied orbitals is localized on two sites. The optimal results are obtained using DOCI or oo-p-CCD orbitals, which give essentially the same results. It is important to note that the optimal DOCI and oo-p-CCD orbitals are localized in nature.

We next wish to explore the contributions of different seniority sectors to the CCD correlation energy in different orbital bases. As a case study, we again use the 6-site Hubbard model at half filling. We plot the correlation energy of p-CCD, which is the seniority zero part of CCD not coupled to the other seniority sectors. In addition, we plot the contributions of the different seniority sectors to the standard CCD correlation energy, which generally includes seniorities zero, two, and four; their sum gives the total CCD correlation. We will start by examining the seniority profile of DOCI orbitals, shown in Figure 3.

With the DOCI optimized orbitals, the main contribution to the CCD correlation energy is from the seniority zero sector. The seniority two sector does not contribute energetically and seniority four contributions are small; they give $6 \%$ of the total energy at $U=1$ and $0.2 \%$ at $U=9$. Interestingly, even for large values of $U$, where the CCD correlation energy is solely due to seniority zero, it still differs from the p-CCD correlation energy. In other words, coupling between the different seniority sectors plays an important role in the amplitude equations. This coupling is apparently responsible for the breakdown of CCD at large values of $U$, since p-CCD (i.e., seniority zero not coupled to the other seniority sectors) does not overcorrelate. Results with oo-p-CCD orbitals are essentially the same and yield the same picture (data not shown).

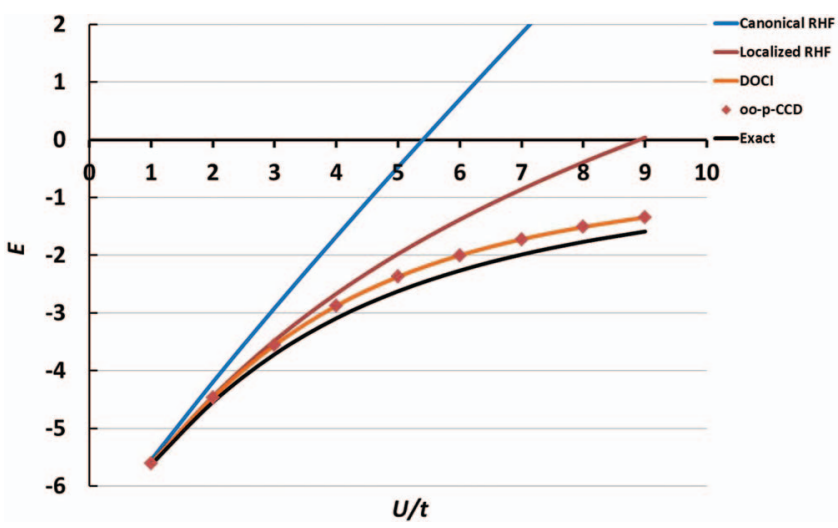

FIG. 2. Performance of p-CCD with different orbital basis sets, compared to the exact result for the 6-site Hubbard chain at half filling.

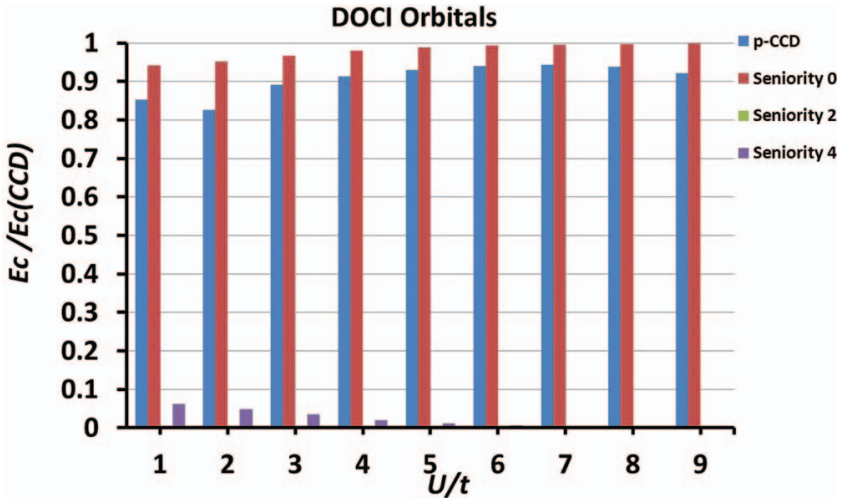

FIG. 3. Contributions from different seniority sectors to the CCD correlation energy for the 6-site Hubbard chain at half-filling with DOCI optimal orbitals.

The upper panel of Figure 4 shows results from the canonical HF basis. There are several prominent differences compared to the DOCI/oo-p-CCD results. First we see that we get contributions from all seniority sectors. While the seniority two contributions are small, seniority four is quite significant. Moreover, one can see that as $U$ gets larger, the p-CCD recovers a smaller fraction of the CCD correlation energy; the ratio between the p-CCD and CCD correlation energies starts at $40 \%$ for $U=1$ and is slightly above $20 \%$ for $U=9$. The seniority zero part of the CCD correlation energy is a little less than $50 \%$ of the total correlation energy at $U=1$ and is still $43 \%$ at $U=9$. For small values of $U$, the contributions from seniority zero and seniority four are comparable, but as $U$ gets larger, so does the seniority four contribution.
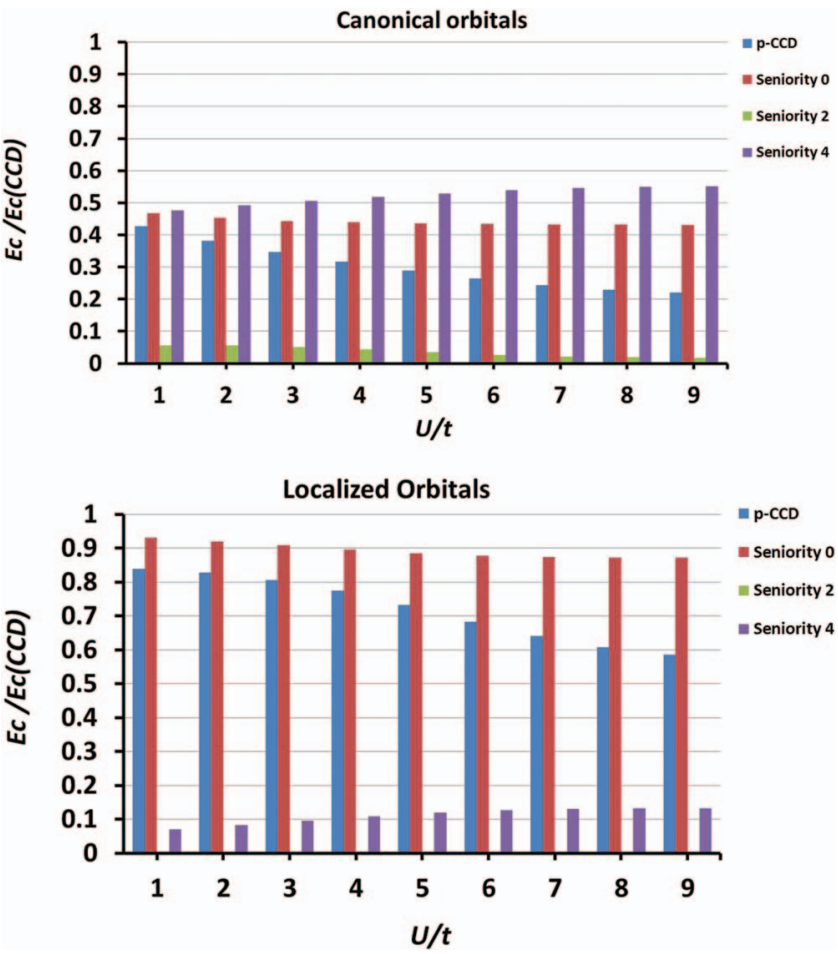

FIG. 4. Contributions from different seniority sectors to the CCD correlation energy for the 6-site Hubbard chain at half filling. Upper panel: Canonical HF orbitals. Lower panel: orbital localization on top of the canonical HF basis. 
Contributions from seniority two are small in this system, and get even smaller as $U$ gets larger.

As discussed above, orbital localization plays an essential role, so we next analyze the localized orbital basis. It is important to bear in mind that CCD is invariant to rotations among the occupied orbitals and among the virtual orbitals, so the CCD correlation energies are the same in the canonical and the localized bases. However, p-CCD is strongly affected by mixing within the occupied or virtual orbitals, as one can see from the lower panel of Figure 4; the effect of mixing occupied and virtual orbitals will be discussed later. At $U=1$, pCCD captures over $80 \%$ of the correlation energy (this should be compared to $40 \%$ in the canonical basis). This goes down to $59 \%$ at $U=9$. A prominent difference is that when using localized orbitals, the dominant contribution to CCD arises from the seniority zero sector. Another difference between the two orbital sets is that in the canonical HF basis, the different amplitudes have the same sign, while in the localized basis we get seniority two and four with both positive and negative signs. Seniority two contributions are individually small with localized orbitals and tend to cancel against each other so that the total contribution of the seniority two sector to the CCD correlation energy is insignificant. As with the canonical HF orbitals, the contributions from seniority four increase as $U$ increases; however, they are significantly smaller (7\% at $U=1$ and $13 \%$ at $U=9$ ). Although we have cancelation effects within the seniority four sector as well, the effect is smaller than in the seniority two sector.

\section{AN ALTERNATIVE PAIRING SCHEME - p-BCCD WITH LOCALIZATION}

Orbital optimization has been well studied for the coupled cluster wave function. ${ }^{1,38,40,51,52}$ As already mentioned the CCD method is invariant to mixing the occupied orbitals or the virtual orbitals; however, it is not invariant to the choice of the reference determinant (mixing between occupied and virtual orbitals). From the Thouless theorem, we know that different reference determinants are related by ${ }^{53}$ $|0\rangle=\exp T_{1}|\Theta\rangle$. In other words, the singles amplitudes of CCSD are responsible for orbital relaxation effects. Single excitations can be excluded by orbital optimization at the CCD level, as done by Scuseria et al. who showed that with CCD optimal orbitals the contributions from $\mathrm{T}_{1}$ are generally small. $^{38}$

Alternatively, one can choose a reference determinant in which all the single excitation amplitudes are zero. As Brueckner orbitals are the set of orbitals in which at FCI all the contributions from single excitations are zero, one obtains approximate Brueckner orbitals at the CCD level by adjusting the reference determinant iteratively such that the solution of the $\mathrm{T}_{1}$ equations is $\mathrm{T}_{1}=0 .{ }^{54}$ This method is known as Brueckner CCD (BCCD). ${ }^{55,56}$ Studies have shown that the energies and properties obtained from BCCD and CCSD are similar, ${ }^{57,58}$ even though the Brueckner orbitals are shown to perform better than Hartree-Fock when dealing with broken symmetry solutions. $.52,59$

We define a one-body Brueckner effective Hamiltonian $\tilde{F}$, whose occupied-occupied and virtual-virtual blocks are,

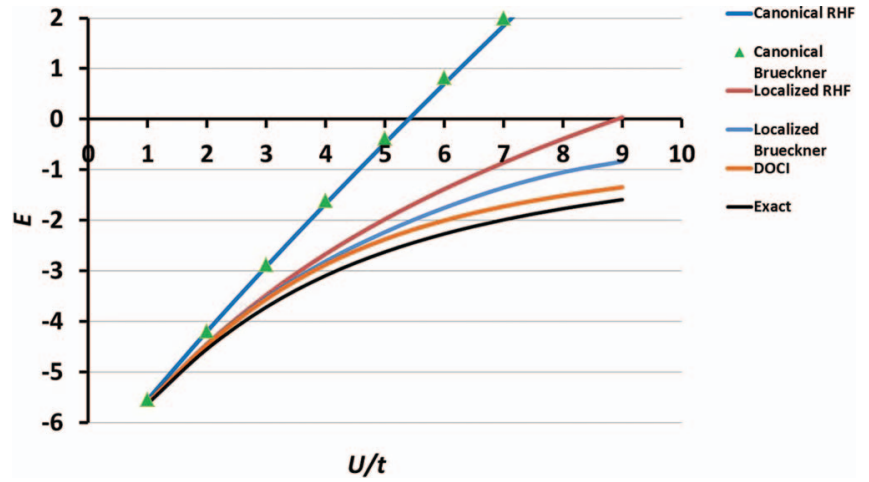

FIG. 5. Performance of p-CCD with canonical Brueckner and localized Brueckner orbitals compared to other methods discussed in the text for the 6-site Hubbard chain at half filling.

respectively,

$$
\begin{aligned}
& \tilde{F}_{i}^{k}=F_{i}^{k}+\sum_{c, d, l} \frac{1}{2} \bar{v}_{c d}^{k l} t_{i l}^{c d}, \\
& \tilde{F}_{c}^{a}=F_{c}^{a}-\sum_{k, l, d} \frac{1}{2} \bar{v}_{c d}^{k l} t_{k l}^{a d},
\end{aligned}
$$

while the occupied-virtual and virtual-occupied blocks are the $T_{1}$ equations. Here, $F$ is the Fock operator and $\bar{v}_{c d}^{k l}=\langle k l \| c d\rangle$ is an antisymmetrized two-electron integral in Dirac's notation. Orbitals $i, k$, and $l$ are occupied, while $a, c$, and $d$ are virtual. The occupied-virtual and virtual-occupied blocks of this Hamiltonian vanish at self-consistency, as it is set to the $\mathrm{T}_{1}$ equation. When we refer to the Brueckner orbitals we refer to the canonical Brueckner orbitals which diagonalize the Hermitian part of the Brueckner effective Hamiltonian.

Figure 5 shows the performance of p-CCD with the different orbital bases discussed in the text and with the canonical and localized Brueckner orbitals. The canonical Brueckner orbitals give results with quality similar to HF canonical orbitals, however, the localized Brueckner orbitals improve over the localized HF orbitals.

These considerations suggest the possibility of an alternative way to obtain a well-defined and nearly optimal pairing scheme for $\mathrm{p}-\mathrm{CCD}$. If one simply diagonalizes the Brueckner effective Hamiltonian formed from the seniority zero $\mathrm{T}_{2}$ amplitudes in what we call pair-BCCD (p-BCCD), one obtains quite poor results. This is simply because the canonical Brueckner orbitals do not give a useful pairing scheme. As we and others have observed, ${ }^{28,60}$ orbital localization is important in p-CCD, and should be incorporated also in any Brueckner-based approach. One can do a single shot localization after the convergence of the p-BCCD scheme, but we find that the best results are achieved if the localization is done self-consistently. Thus, at each cycle, after diagonalization of the Brueckner effective Hamiltonian, we localize the occupied and virtual orbitals. We refer to this approach as localized p-BCCD. We note that convergence of the Brueckner determinant is easier to achieve than convergence of the pairing scheme. Even when the Brueckner determinant is converged, the energy is very sensitive to mixing within the occupied or virtual blocks. In other words, although the Brueckner 
TABLE III. Comparison between correlation energies recovered by DOCI and the localized p-BCCD optimization.

\begin{tabular}{|c|c|c|c|c|c|c|}
\hline \multirow[b]{2}{*}{$U$} & \multicolumn{2}{|c|}{6 sites $(\%)$} & \multicolumn{2}{|c|}{8 sites $(\%)$} & \multicolumn{2}{|c|}{10 sites $(\%)$} \\
\hline & $\mathrm{Ec}(\mathrm{DOCI})$ & Ec(localized p-BCCD) & $\mathrm{Ec}(\mathrm{DOCI})$ & Ec(localized p-BCCD) & $\mathrm{Ec}(\mathrm{DOCI})$ & Ec(localized p-BCCD) \\
\hline 2 & 85.48 & 85.32 & 83.42 & 83.25 & 82.00 & 81.84 \\
\hline 5 & 92.02 & 91.86 & 90.75 & 90.57 & 89.92 & 89.73 \\
\hline 9 & 96.97 & 96.74 & 96.51 & 96.25 & 96.23 & 95.94 \\
\hline
\end{tabular}

determinant itself is invariant to mixing occupied orbitals with each other, $\mathrm{p}-\mathrm{CCD}$ is very sensitive to such mixing (and to virtual-virtual mixing), so that while the Brueckner determinant is converged, the p-CCD energy may not be. We have, therefore, converged the Brueckner determinant (by insisting that $\mathrm{T}_{1}=0$ self-consistently) and also insisted that the energy be converged to a value of $10^{-7}$ in units of the Hubbard parameter $t$.

Table III shows the amount of correlation energy recovered by DOCI and localized p-BCCD for 6-, 8-, and 10-site Hubbard chains at half filling. The localized p-BCCD approach yields results comparable to DOCI and, thus, to oop-CCD.

This approach also works for larger chains. Table IV shows the correlation energy recovered for 14 and 20 sites at half filling. For those systems, we again see the same trends as discussed for the smaller chains and the results are comparable to oo-p-CCD. For 20 sites with only 12 electrons the results are inferior to oo-p-CCD, but still, a significant amount of correlation energy can be recovered.

The important message here is the role of the different components: the Brueckner orbitals rotate the reference determinant and exclude the need for $\mathrm{T}_{1}$ amplitudes. For any pair wave function we additionally need to define the rotation of the occupied and virtual orbitals. The fact that the combination of p-BCCD and orbital localization recovers DOCI results demonstrates again the important role of localization; the energetically significant contributions to the correlation energy need to arise from the seniority zero sector to obtain optimal performance. The localized p-BCCD approach will work well when the optimal pairing is indeed dominated by orbital localization, as is the case for Hubbard chains at half filling.

\section{ACHIEVING BETTER BALANCE WITHIN THE DIFFERENT SENIORITY SECTORS - FREEZING THE PAIR AMPLITUDES}

As the seniority zero sector plays an essential role in the description of static correlation, it seems natural to solve

TABLE IV. Performance of the localized p-BCCD optimization for 14- and 20-site Hubbard chains at half filling, and 20 sites away from half-filling.

\begin{tabular}{cccc}
\hline \hline$U$ & 14 sites 14 e (\%) & 20 sites 20 e (\%) & 20 sites 12 e (\%) \\
\hline 2 & 79.97 & & 69.11 \\
5 & 88.69 & 87.86 & 76.26 \\
9 & 95.58 & 95.30 & 84.15 \\
\hline
\end{tabular}

first the seniority zero sector, and then account for residual dynamic correlation with the other seniority sectors without changing the seniority zero wave function amplitudes. In our attempts to achieve an improved description at the CCD level, we have tried freezing the pair amplitudes. In other words, we first solve the p-CCD equations for the seniority zero amplitudes, then with these amplitudes frozen, we solve the usual CCD or CCSD equations only for the seniority two and four amplitudes. We refer to this approach as fp-CCD and fpCCSD (fp for "frozen pair").

Figure 6 shows results for 6- and 8-site Hubbard chains at half filling, with canonical HF orbitals. For the 6-site chain, CCD and CCSD start to overcorrelate at $U=3-4$. As shown earlier, $\mathrm{p}$-CCD gives qualitatively wrong results in this orbital basis, and significantly undercorrelates. This effect is significantly moderated in fp-CCD and fp-CCSD. For comparison purposes, we have added DOCI results. Surprisingly, freezing the pair amplitudes obtained in the canonical Hartree-Fock basis, one achieves a much more balanced description. fpCCD and fp-CCSD follow DOCI up to $U=4$, and for larger $U$ they are above DOCI (in other words, they do not overcorrelate and while results are only of moderate quality, the
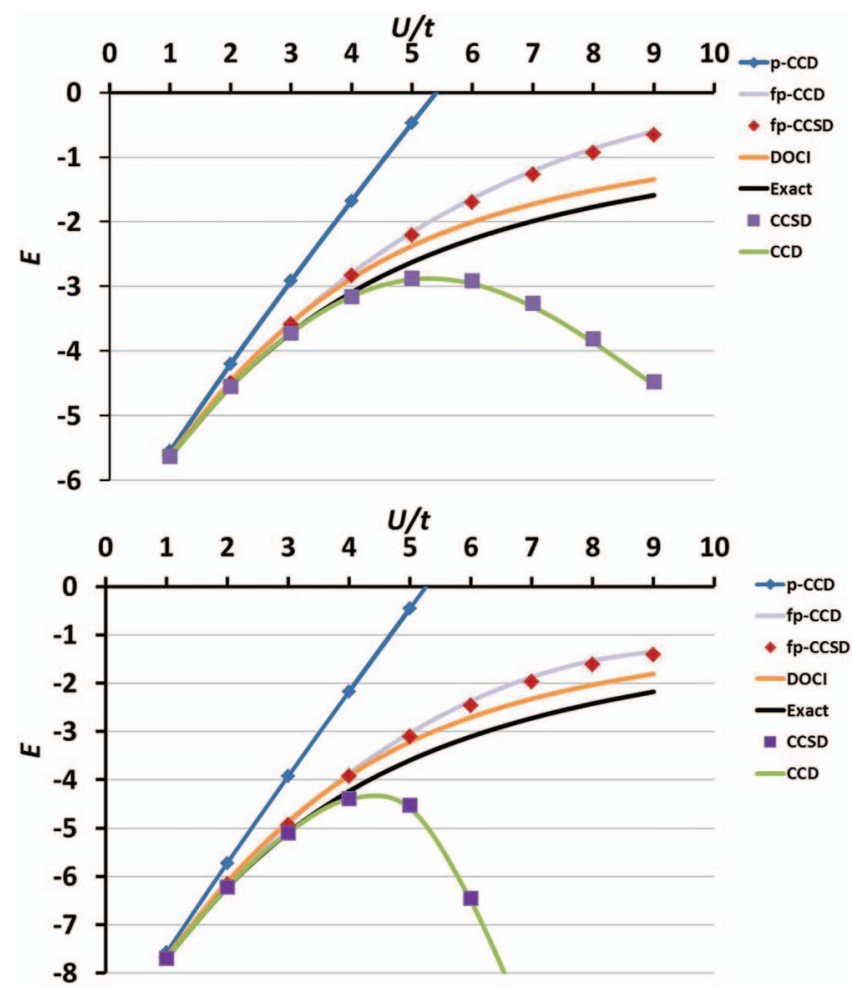

FIG. 6. Upper panel: results for the 6-site Hubbard chain at half-filling. Lower panel: results for the 8-site Hubbard chain at half-filling. 


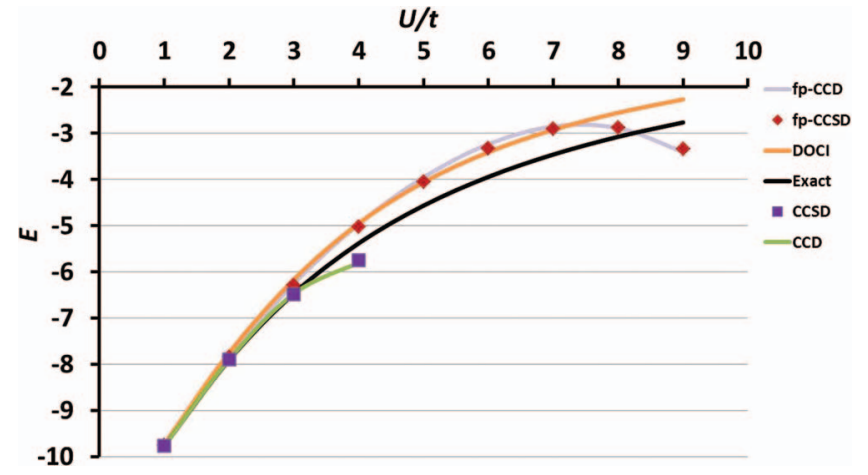

FIG. 7. 10-site Hubbard chain at half-filling with different methods.

method does not break down catastrophically). In this basis, the description with the frozen pairs is much more balanced than p-CCD, CCD, or CCSD. Results for the 8-site chain are shown at the lower panel of Figure 6. Here, again CCD and CCSD start to overcorrelate near $U=4$, although their deterioration seems more severe than in the 6-site case. Again, p-CCD gives a straight line in the canonical HF basis, while fp-CCD and fp-CCSD follow the DOCI curve and are above it after $U=5$.

Figure 7 shows the results for the 10-site chain at halffilling. We were unable to converge CCD or CCSD for $U>4$. We again see the same trend for fp-CCD and fp-CCSD; they follow the DOCI curve for some time, and then diverge from it. Unlike for smaller systems, they eventually begin to overcorrelate, but where CCD and CCSD start to overcorrelate near $U=4$, the frozen pair methods do not begin to break down until $U \approx 8$.

These results are intriguing, since p-CCD in the canonical Hartree-Fock basis does not correctly describe static correlation. The coupling between the different seniority sectors is apparently unbalanced for strongly correlated systems, and simply by constraining the pair amplitudes we can get a better description. In Figure 8, we show the different seniority contributions to the correlation energy of fp-CCD for our test case of the 6-site chain at half filling. We see here, that unlike the canonical HF basis without frozen pairs, the seniority two contributions are fairly constant, and that the differences

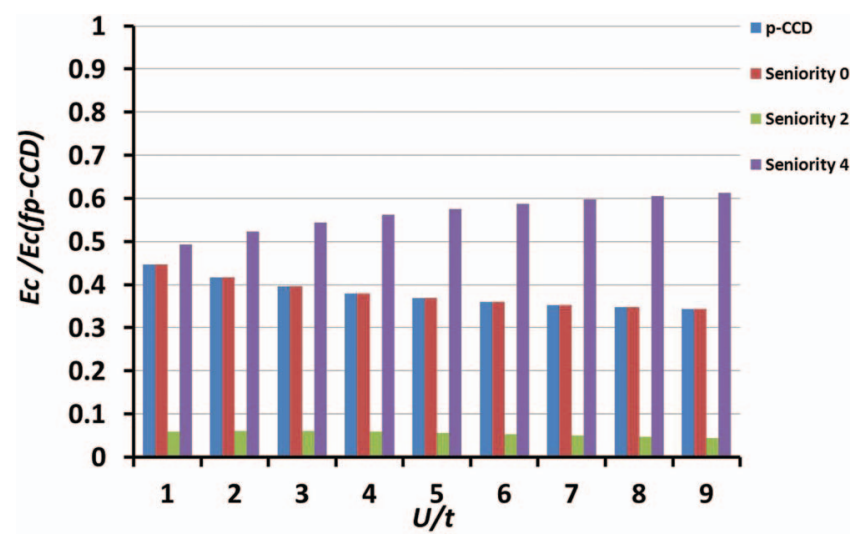

FIG. 8. Different seniority contributions for the 6-site Hubbard chain at half filling calculated with fp-CCD. between contributions from seniority zero and four become more pronounced as $U$ gets larger.

\section{CONCLUSIONS}

We have focused on the seniority zero pair CCD theory. While CCD and CCSD are essentially limited to the description of weak correlation, one can describe strong correlation effects using only the seniority zero sector of CCD. Even though the method is not invariant with respect to orbital rotations, it is perfectly well-defined by the basis that makes the CC energy stationary with respect to all orbital rotations. The optimal orbitals turn out to be localized, and the resulting seniority zero sector is the dominant contribution to the correlation energy.

Understanding the role of different seniority sectors with different orbitals gives a clearer insight regarding the optimal pairing scheme. A nearly optimal pairing scheme can be obtained using the Brueckner orbitals for p-CCD with localization. The fact that CCD is known to capture dynamic correlation, while its seniority zero part can capture the static correlation, is intriguing, and suggests an imbalance between the different seniority sectors at the CCD level. One can obtain a better description by solving p-CCD equations and freezing the seniority zero amplitudes in the CCD equations. We have demonstrated that this postpones the breakdown of $\mathrm{CCD}$ as the system gets strongly correlated.

\section{ACKNOWLEDGMENTS}

The authors thank Jorge Dukelsky for useful discussions, and the authors of Ref. 31 for providing us their orbital optimization data for numerical comparison of results. This work was supported by the Department of Energy, Office of Basic Energy Sciences, Grant No. DE-FG02-09ER16053. G.E.S. is a Welch Foundation Chair (C-0036). T.S. is an Awardee of the Weizmann Institute of Science - National Postdoctoral Award Program for Advancing Women in Science.

${ }^{1}$ G. D. Purvis and R. J. Bartlett, J. Chem. Phys. 76, 1910 (1982).

${ }^{2}$ K. Raghavachari, G. W. Trucks, J. A. Pople, and M. Head-Gordon, Chem. Phys. Lett. 157, 479 (1989).

${ }^{3}$ R. J. Bartlett and M. Musial, Rev. Mod. Phys. 79, 291 (2007).

${ }^{4}$ T. D. Crawford and H. F. Schaefer III, Rev. Comput. Chem. 14, 33 (2000).

${ }^{5}$ W. Kutzelnigg, Theor. Chim. Acta 68, 445 (1985).

${ }^{6}$ N. Oliphant and L. Adamowicz, J. Chem. Phys. 95, 6645 (1991).

${ }^{7}$ G. E. Scuseria and H. F. Schaefer III, Chem. Phys. Lett. 152, 382 (1988).

${ }^{8}$ J. Noga and R. J. Bartlett, J. Chem. Phys. 86, 7041 (1987).

${ }^{9}$ B. O. Roos, in Advances in Chemical Physics (John Wiley \& Sons, Inc., 2007), p. 399.

${ }^{10}$ K. Ruedenberg, M. W. Schmidt, M. M. Gilbert, and S. T. Elbert, Chem. Phys. 71, 41 (1982).

${ }^{11}$ C. A. Jiménez-Hoyos, T. M. Henderson, T. Tsuchimochi, and G. E. Scuseria, J. Chem. Phys. 136, 164109 (2012).

${ }^{12}$ G. E. Scuseria, C. A. Jiménez-Hoyos, T. M. Henderson, K. Samanta, and J. K. Ellis, J. Chem. Phys. 135, 124108 (2011).

${ }^{13}$ J. K. Ellis, R. L. Martin, and G. E. Scuseria, J. Chem. Theor. Comput. 9, 2857 (2013).

${ }^{14}$ W. Kutzelnigg, Chem. Phys. 401, 119 (2012).

${ }^{15}$ W. Kutzelnigg, J. Phys. Chem. A 114, 8913 (2010).

${ }^{16}$ D. I. Lyakh, M. Musial, V. F. Lotrich, and R. J. Bartlett, Chem. Rev. 112, 182 (2012).

${ }^{17}$ K. Jankowski, J. Paldus, I. Grabowski, and K. Kowalski, J. Chem. Phys. 97, 7600 (1992). 
${ }^{18} \mathrm{X}$. Li and J. Paldus, J. Chem. Phys. 119, 5320 (2003).

${ }^{19}$ O. Hino, T. Kinoshita, G. K.-L. Chan, and R. J. Bartlett, J. Chem. Phys. 124, 114311 (2006).

${ }^{20}$ D. I. Lyakh, V. V. Ivanov, and L. Adamowicz, J. Chem. Phys. 122, 024108 (2005).

${ }^{21}$ F. A. Evangelista and J. Gauss, J. Chem. Phys. 134, 114102 (2011).

${ }^{22}$ U. S. Mahapatra, B. Datta, and D. Mukherjee, J. Chem. Phys. 110, 6171 (1999).

${ }^{23}$ S. Das, M. Kállay, and D. Mukherjee, Chem. Phys. 392, 83 (2012).

${ }^{24}$ T. Van Voorhis and M. Head-Gordon, J. Chem. Phys. 113, 8873 (2000).

${ }^{25}$ F. A. Evangelista, J. Chem. Phys. 134, 224102 (2011).

${ }^{26}$ D. W. Small and M. Head-Gordon, Phys. Chem. Chem. Phys. 13, 19285 (2011).

${ }^{27}$ D. W. Small and M. Head-Gordon, J. Chem. Phys. 137, 114103 (2012).

${ }^{28}$ L. Bytautas, T. M. Henderson, C. A. Jiménez-Hoyos, J. K. Ellis, and G. E. Scuseria, J. Chem. Phys. 135, 044119 (2011).

${ }^{29} \mathrm{P}$. Ring and P. Schuck, The Nuclear Many Body Problem (Springer, Berlin, 2000).

${ }^{30}$ P. A. Limacher, T. D. Kim, P. W. Ayers, P. A. Johnson, S. De Baerdemacker, D. Van Neck, and P. Bultinck, Mol. Phys. 112, 853 (2014).

${ }^{31}$ K. Boguslawski, P. Tecmer, P. W. Ayers, P. Bultinck, S. De Baerdemacker, and D. Van Neck, Phys. Rev. B 89, 201106 (2014).

${ }^{32}$ P. Tecmer, K. Boguslawski, P. A. Johnson, P. A. Limacher, M. Chan, T. Verstraelen, and P. W. Ayers, "Assessing the accuracy of new geminal-based approaches," J. Phys. Chem. A (published online).

${ }^{33}$ K. Boguslawski, P. Tecmer, P. A. Limacher, P. A. Johnson, P. W. Ayers, P. Bultinck, S. De Baerdemacker, and D. Van Neck, Phys. Rev. B 89, 201106 (2014).

${ }^{34}$ P. A. Limacher, P. W. Ayers, P. A. Johnson, S. De Baerdemacker, D. Van Neck, and P. Bultinck, J. Chem. Theor. Comput. 9, 1394 (2013).

${ }^{35}$ P. A. Johnson, P. W. Ayers, P. A. Limacher, S. De Baerdemacker, D. Van Neck, and P. Bultinck, Comput. Theor. Chem. 1003, 101 (2013).

${ }^{36}$ P. R. Surjan, A. Szabados, P. Jeszenszki, and T. Zoboki, J. Math. Chem. 50, 534 (2012).

${ }^{37}$ J. A. Parkhill and M. Head-Gordon, J. Chem. Phys. 133, 124102 (2010).

${ }^{38}$ G. E. Scuseria and H. F. Schaefer III, Chem. Phys. Lett. 142, 354 (1987).

${ }^{39}$ E. A. Salter, G. W. Trucks, and R. J. Bartlett, J. Chem. Phys. 90, 1752 (1989).

${ }^{40}$ U. Bozkaya, J. M. Turney, Y. Yamaguchi, H. F. Schaefer III, and C. D. Sherrill, J. Chem. Phys. 135, 104103 (2011).
${ }^{41}$ J. Hubbard, Proc. R. Soc. London, Ser. A 276, 238 (1963).

${ }^{42}$ H. Bethe, Z. Phys. 71, 205 (1931).

${ }^{43}$ E. H. Lieb and F. Y. Wu, Phys. Rev. Lett. 20, 1445 (1968).

${ }^{44}$ S. R. White, Phys. Rev. Lett. 69, 2863 (1992).

${ }^{45}$ U. Schollwöck, Rev. Mod. Phys. 77, 259 (2005).

${ }^{46}$ B. Bauer, L. D. Carr, H. G. Evertz, A. Feiguin, J. Freire, S. Fuchs, L. Gamper, J. Gukelberger, E. Gull, S. Guertler, A. Hehn, R. Igarashi, S. V. Isakov, D. Koop, P. N. Ma, P. Mates, H. Matsuo, O. Parcollet, G. Pawlowski, J. D. Picon, L. Pollet, E. Santos, V. W. Scarola, U. Schollwock, C. Silva, B. Surer, S. Todo, S. Trebst, M. Troyer, M. L. Wall, P. Werner, and S. Wessel, J. Stat. Mech.: Theory Exp. 2011, P05001.

${ }^{47}$ A. F. Albuquerque, F. Alet, P. Corboz, P. Dayal, A. Feiguin, S. Fuchs, L. Gamper, E. Gull, S. Gürtler, A. Honecker, R. Igarashi, M. Körner, A. Kozhevnikov, A. Läuchli, S. R. Manmana, M. Matsumoto, I. P. McCulloch, F. Michel, R. M. Noack, G. Pawłowski, L. Pollet, T. Pruschke, U. Schollwöck, S. Todo, S. Trebst, M. Troyer, P. Werner, and S. Wessel, J. Magn. Magn. Mater. 310, 1187 (2007).

${ }^{48}$ G. E. Scuseria, A. C. Scheiner, J. E. Rice, T. J. Lee, and H. F. Schaefer III, Int. J. Quantum Chem. 32, 495 (1987).

${ }^{49}$ A. C. Scheiner, G. E. Scuseria, J. E. Rice, T. J. Lee, and H. F. Schaefer III, J. Chem. Phys. 87, 5361 (1987).

${ }^{50}$ C. Edmiston and K. Ruedenberg, Rev. Mod. Phys. 35, 457 (1963).

${ }^{51}$ A. I. Krylov, C. D. Sherrill, E. F. C. Byrd, and M. Head-Gordon, J. Chem. Phys. 109, 10669 (1998)

${ }^{52}$ C. D. Sherrill, A. I. Krylov, E. F. C. Byrd, and M. Head-Gordon, J. Chem. Phys. 109, 4171 (1998).

${ }^{53}$ D. J. Thouless, The Quantum Mechanics of Many Body Systems (Academic, New York, 1961)

${ }^{54}$ G. E. Scuseria, Int. J. Quantum Chem. 55, 165 (1995).

${ }^{55}$ C. Hampel, K. A. Peterson, and H.-J. Werner, Chem. Phys. Lett. 190, 1 (1992).

${ }^{56}$ J. F. Stanton, J. Gauss, and R. J. Bartlett, J. Chem. Phys. 97, 5554 (1992).

${ }^{57}$ T. J. Lee, R. Kobayashi, N. C. Handy, and R. D. Amos, J. Chem. Phys. 96, 8931 (1992).

${ }^{58}$ R. Kobayashi, H. Koch, P. Jørgensen, and T. J. Lee, Chem. Phys. Lett. 211, 94 (1993).

${ }^{59}$ W. D. Allen, D. A. Horner, R. L. Dekock, R. B. Remington, and H. F. Schaefer III, Chem. Phys. 133, 11 (1989).

${ }^{60}$ P. R. Surjan, Correlation and Localization (Springer, Berlin, 1999). 